\title{
PERCEPÇÕES DE PROFESSORES QUANTO À INTRODUÇÃO DE BIM NO CURRÍCULO ${ }^{1}$
}

\author{
ATTITUDES OF LECTURERS TO THE INTRODUCTION OF BIM INTO THE \\ CURRICULUM
}

\author{
Maria Bernardete Barison \\ Universidade Estadual de Londrina, UEL \\ barison@uel.br \\ Eduardo Toledo Santos \\ Universidade de São Paulo, USP \\ etoledo@usp.br
}

\begin{abstract}
RESUMO
Dada a importância de ensinar Modelagem da Informação da Construção (Building Information Modeling - BIM) para futuros arquitetos e engenheiros civis, várias universidades têm demonstrado interesse em introduzir este assunto no currículo. No entanto, como se trata de um assunto relativamente novo, ainda não está claro para todos, como este tema deve ser abordado no contexto universitário. Algumas universidades têm adotado a estratégia de conduzir pesquisas para saber a opinião de professores, alunos e profissionais, como subsídio às suas decisões nesse sentido. Inicialmente, este trabalho apresenta uma revisão da literatura sobre projetos piloto que algumas instituições acadêmicas têm desenvolvido, para implantação de BIM em seus currículos. Com base nas informações levantadas na etapa de revisão da literatura, foi realizada uma investigação com professores de dois cursos de uma universidade pública brasileira, para conhecer suas percepções quanto ao ensino de BIM. A investigação incluiu a análise dos curriculos e o envio de um questionário online para os professores. Os resultados são apresentados, discutidos e, ao final, é apresentada uma proposta para a reformulação dos currículos que contempla as sugestões dos professores. Assim, o objetivo deste trabalho é oferecer algumas diretrizes para universidades que desejam implementar BIM nos currículos de seus cursos, baseadas na literatura e levando em conta a percepção dos docentes.
\end{abstract}

Palavras-chave: Currículo. Engenharia Civil. Arquitetura. Modelagem da Informação da Construção. BIM.

\begin{abstract}
Owing to the importance of teaching Building Information Modeling (BIM) to future architects and civil engineers, several universities have shown an interest in introducing this subject in the curriculum. However, as BIM is a relatively new subject, it is not yet clear to everyone how this subject should be regarded. Some universities have adopted a strategy of conducting research to find out the opinions of lecturers, students and professionals, so that their decisions can have academic support. This study includes a literature review on the pilot projects that some academic institutions have carried out before introducing BIM into their curriculum. On the basis of information gathered from the literature review, an investigation was carried out into the views of lecturers who were running two courses at a Brazilian public university to find out their attitudes to BIM education. This research included an analysis of the curriculum and an online questionnaire which was answered by the lecturers. The results were examined and discussed and in the end a plan was put forward for a revised curriculum which incorporated some
\end{abstract}

IBARISON, M. B.; SANTOS, E. T. Percepções de professores quanto à introdução de BIM no currículo. In: ENCONTRO BRASILEIRO DE TECNOLOGIA DE INFORMAÇÃO E COMUNICAÇÃO NA CONSTRUÇÃO, 7., 2015, Recife. Anais... Porto Alegre: ANTAC, 2015. 
of the suggestions made by the lecturers. The purpose of this study is to provide some guidelines for universities that wish to implement BIM in the curriculum of their courses that are based on the literature and take into account the attitudes of the lecturers.

Keywords: Curriculum. Civil Engineering. Architecture. Building Information Modeling. BIM.

\section{INTRODUÇÃO}

Este artigo apresenta resultados parciais de uma pesquisa de doutorado sobre BIM e Educação. O objetivo do artigo é oferecer algumas diretrizes para universidades que desejam implementar BIM nos currículos de seus cursos. Estas diretrizes são baseadas em revisão da literatura sobre projetos piloto que algumas escolas têm desenvolvido para implantar BIM no currículo, assim como, em resultados de uma pesquisa com professores de dois cursos de uma universidade pública brasileira sobre suas percepções em relação a BIM. Ementas de disciplinas com potencial para introduzir BIM foram analisadas e em seguida foi enviado um questionário online para os professores dessas disciplinas. Os professores deram suas opiniões sobre questões relacionadas ao BIM discutidas atualmente na literatura. Os resultados do questionário são apresentados, assim como, uma proposta para reformulação dos currículos desses dois cursos e levando em conta a percepções dos docentes.

\section{REVISÃO DA LITERATURA}

Antes de introduzir BIM no currículo, algumas escolas preferem fazer um projeto-piloto, no qual são definidas algumas estratégias. Barison (2015) identificou quatro tipos de estratégias: (a) entrevistar profissionais da indústria para obter sugestões sobre conteúdos, avaliação, assim como para validação das propostas; (b) entrevistar professores e alunos para conhecer suas percepções em relação ao BIM; (c) analisar ementas de disciplinas BIM de outras escolas e (d) construir uma lista de competências BIM.

Após obter estas informações, os três próximos passos são: (a) selecionar disciplinas do currículo em que BIM pode ser introduzido, assim como planejar novas disciplinas; (b) definir os objetivos da implantação de BIM na disciplina (se deverá ser ensinada uma ferramenta BIM ou se BIM será um facilitador para os alunos entenderem conteúdos da disciplina; e (c) definir o nível de proficiência BIM (NPBIM) da disciplina, proposto por Barison (2015), ou a etapa do ensino de BIM (ilustração, manipulação, aplicação ou colaboração) proposta por MacDonald (2011).

De acordo com Gao, McIntyre e Ge (2012) também é necessário definir a habilidade do professor, as mudanças que a disciplina irá requerer e os métodos de avaliação. Ao final, os resultados do estudo devem ser relatados aos integrantes do curso.

Pikas, Sacks e Hazzan (2013) recomendam que, após efetuar as mudanças na disciplina, os resultados devem ser monitorados e medidos conforme alguns parâmetros e que as disciplinas sejam revisadas e melhoradas continuamente. Porém, se BIM já foi implementado, a escola deveria analisar o currículo para saber o que realmente precisa ser ensinado. O Quadro 1 apresenta alguns exemplos de projetos-piloto (BARISON, 2015).

Quadro 1 - Projetos piloto para introdução de BIM no currículo

\begin{tabular}{|l|l|l|}
\hline Referência & Objetivo & Estratégias \\
\hline $\begin{array}{l}\text { Mutai e Guidera } \\
(2008)\end{array}$ & $\begin{array}{l}\text { Fornecer recomendações para o } \\
\text { ensino de BIM. }\end{array}$ & $\begin{array}{l}\text { Pesquisar em construtoras o nível de uso } \\
\text { de BIM. }\end{array}$ \\
\hline $\begin{array}{l}\text { Schmelter e } \\
\text { Cory (2009) }\end{array}$ & $\begin{array}{l}\text { Planejar uma disciplina BIM para o } \\
\text { curso de Tecnologia de } \\
\text { Computação Gráfica. }\end{array}$ & $\begin{array}{l}\text { Definir conteúdos. Enviar questionário para } \\
\text { os profissionais. }\end{array}$ \\
\hline
\end{tabular}




\begin{tabular}{|c|c|c|}
\hline $\begin{array}{l}\text { Taylor, Liu e } \\
\text { Hein (2008) }\end{array}$ & $\begin{array}{l}\text { Planejar uma disciplina eletiva de } \\
\text { introdução ao BIM para cursos de } \\
\text { Arquitetura e Gestão da } \\
\text { Construção. }\end{array}$ & $\begin{array}{l}\text { Definir conteúdos e dialogar com } \\
\text { construtoras que estão usando BIM. }\end{array}$ \\
\hline $\begin{array}{l}\text { Gordon, } \\
\text { Azambuja e } \\
\text { Werner (2009) }\end{array}$ & $\begin{array}{l}\text { Preparar uma disciplina introdutória } \\
\text { BIM e ensinar BIM em várias } \\
\text { disciplinas de Gestão da } \\
\text { Construção. }\end{array}$ & $\begin{array}{l}\text { Definir, nas disciplinas selecionadas, se } \\
\text { BIM será um facilitador da aprendizagem } \\
\text { ou um conteúdo da disciplina. }\end{array}$ \\
\hline $\begin{array}{l}\text { Clevengeret al. } \\
(2010)\end{array}$ & $\begin{array}{l}\text { Preparar uma disciplina introdutória } \\
\text { BIM e módulos interativos para o } \\
\text { curso de Gestão da Construção }\end{array}$ & Consultar profissionais. \\
\hline $\begin{array}{l}\text { Wong, Wong e } \\
\text { Nadeem (2011) }\end{array}$ & $\begin{array}{l}\text { Ensinar BIM em várias disciplinas } \\
\text { do curso 'Building and Real Estate'. }\end{array}$ & $\begin{array}{l}\text { Entrevistar professores e alunos da } \\
\text { universidade. }\end{array}$ \\
\hline $\begin{array}{l}\text { Cooksey e Schiff } \\
(2012)\end{array}$ & $\begin{array}{l}\text { Investigar se é importante ensinar } \\
\text { conteúdos BIM para alunos de } \\
\text { Engenharia Civil. }\end{array}$ & $\begin{array}{l}\text { Revisar a literatura, aplicar questionários e } \\
\text { entrevistas com empresas. }\end{array}$ \\
\hline Bernstein (2011) & $\begin{array}{l}\text { Um modelo útil para entender como } \\
\text { o currículo de arquitetura tem } \\
\text { evoluído e pode ser transformado } \\
\text { com BIM. }\end{array}$ & $\begin{array}{l}\text { Baseado no trabalho de Nicholas } \\
\text { Negroponte (Achitecture Machine) o } \\
\text { currículo de arquitetura é analisado a partir } \\
\text { das fases: acomodação, adaptação e } \\
\text { evolução. }\end{array}$ \\
\hline $\begin{array}{l}\text { MacDonald } \\
(2012)\end{array}$ & $\begin{array}{l}\text { Reformular disciplinas de currículos } \\
\text { de escolas na Austrália. }\end{array}$ & $\begin{array}{l}\text { Introduzir BIM no currículo seguindo as } \\
\text { etapas: ilustração, manipulação, aplicação } \\
\text { e colaboração. }\end{array}$ \\
\hline $\begin{array}{l}\text { Nejat, Darwish e } \\
\text { Ghebrab (2012) }\end{array}$ & $\begin{array}{l}\text { Propor uma ementa BIM de acordo } \\
\text { com a perspectiva da academia. }\end{array}$ & $\begin{array}{l}\text { Definir um questionário, testá-lo e enviá-lo } \\
\text { para uma associação de construtores. }\end{array}$ \\
\hline $\begin{array}{l}\text { Lee e Hollar } \\
(2013)\end{array}$ & $\begin{array}{l}\text { Introduzir BIM no Ensino de Gestão } \\
\text { da Construção. }\end{array}$ & $\begin{array}{l}\text { Aplicar um questionário a especialistas em } \\
\text { BIM/VDC, revisar a literatura e analisar } \\
\text { ementas de disciplinas BIM. }\end{array}$ \\
\hline $\begin{array}{l}\text { Ahn, Cho e Lee } \\
(2013)\end{array}$ & $\begin{array}{l}\text { Desenvolver a disciplina 'BIM in } \\
\text { Construction Management' para } \\
\text { cursos de Gestão da Construção e } \\
\text { Engenharia Civil. }\end{array}$ & $\begin{array}{l}\text { Revisar a literatura, analisar ementas, } \\
\text { entrevistar especialistas e validar a } \\
\text { proposta. }\end{array}$ \\
\hline $\begin{array}{l}\text { Wu e Issa } \\
(2013 a)\end{array}$ & $\begin{array}{l}\text { Facilitar a colaboração entre } \\
\text { academia e indústria. }\end{array}$ & $\begin{array}{l}\text { Enviar questionário on-line para } \\
\text { professores e profissionais. }\end{array}$ \\
\hline $\begin{array}{l}\text { Gao, Mc Intyre e } \\
\text { Ge (2012) }\end{array}$ & $\begin{array}{l}\text { Introduzir BIM no currículo de } \\
\text { Engenharia Civil. }\end{array}$ & $\begin{array}{l}\text { Ensinar BIM em várias disciplinas } \\
\text { introduzindo-o de forma gradual. }\end{array}$ \\
\hline $\begin{array}{l}\text { Sacks e Pikas } \\
(2013)\end{array}$ & $\begin{array}{l}\text { Metodologia para as escolas } \\
\text { definirem conteúdos BIM. }\end{array}$ & $\begin{array}{l}\text { Construir um quadro com } 39 \text { competências } \\
\text { BIM. }\end{array}$ \\
\hline $\begin{array}{l}\text { Pikas, Sacks e } \\
\text { Hazzan (2013) }\end{array}$ & $\begin{array}{l}\text { Analisar se as disciplinas que } \\
\text { ensinam BIM estão desenvolvendo } \\
\text { habilidades BIM. }\end{array}$ & $\begin{array}{l}\text { Analisar o currículo atual, selecionar as } \\
\text { disciplinas com potencial BIM, desenvolver } \\
\text { conteúdos e avaliar os resultados. }\end{array}$ \\
\hline $\begin{array}{l}\text { Mandhar e } \\
\text { Mandhar (2013) }\end{array}$ & $\begin{array}{l}\text { Propor um modelo para introdução } \\
\text { de BIM em currículos de arquitetura } \\
\text { de escolas no Reino Unido. }\end{array}$ & $\begin{array}{l}\text { Seguir três níveis de proficiência BIM: } \\
\text { inicial, transitório e avançado. }\end{array}$ \\
\hline Lucas (2014) & $\begin{array}{l}\text { Desenvolver um plano de } \\
\text { implementação de BIM no currículo. }\end{array}$ & $\begin{array}{l}\text { Revisão da literatura; discussões informais } \\
\text { e entrevistas com profissionais. }\end{array}$ \\
\hline
\end{tabular}

Fonte: Barison (2015, p. 84)

\section{ANÁLISE DE DOIS CURRÍCULOS DE UMA UNIVERSIDADE BRASILEIRA}

Os currículos selecionados para o estudo são dos cursos de Arquitetura e Urbanismo e de Engenharia Civil de uma universidade pública do Estado de São Paulo. Após analisar as ementas das disciplinas dos dois currículos, foram selecionadas as disciplinas consideradas com potencial para a introdução de BIM. O critério utilizado na análise das ementas foi 
'possuir ou não', conteúdos relacionados com CAD, Projeto, Representação Gráfica Digital, Tecnologia da Construção e Gerenciamento de Projeto e Construção. Ao todo foram selecionadas 28 disciplinas do currículo de Arquitetura e Urbanismo e 29 disciplinas do currículo de Engenharia Civil.

\subsection{Percepções de professores quanto ao ensino de BIM}

Para conhecer a percepção de professores quanto ao ensino de BIM, foi aplicado um questionário online com 90 professores que lecionaram, em 2013, as disciplinas consideradas com potencial para a introdução de BIM. O questionário esteve disponível no período 29/03 a 15/04 de 2014 e obteve uma taxa de resposta de $39 \%$, ( $N=35)$, dos quais, $19 \%(\mathrm{~N}=17)$ são professores de Arquitetura e 20\% ( $\mathrm{N}=18)$ são professores de Engenharia Civil, conforme mostra a Figura 1.

Figura 1 - Taxa de respostas obtidas do questionário com os professores

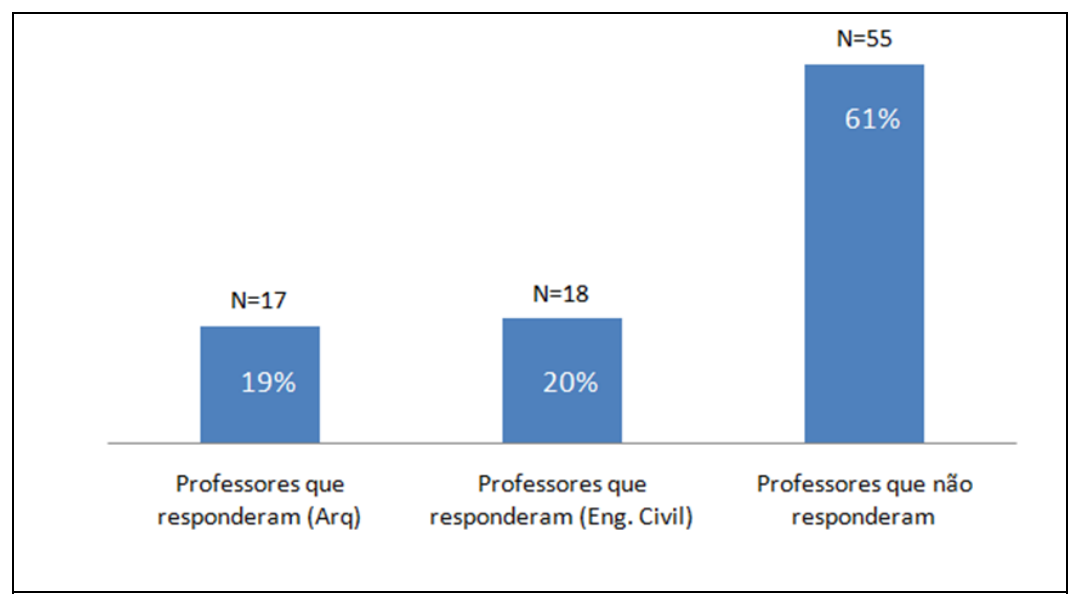

Fonte: Os autores, 2015

A maioria dos respondentes, que corresponde a $83 \%(\mathrm{~N}=29)$ afirma conhecer BIM, porém, apenas $17 \%(\mathrm{~N}=6)$ ensina BIM em sua disciplina, dados que podem ser visualizados na Figura 2.

Figura 2 - Quantidade de professores que conhecem e ensinam BIM

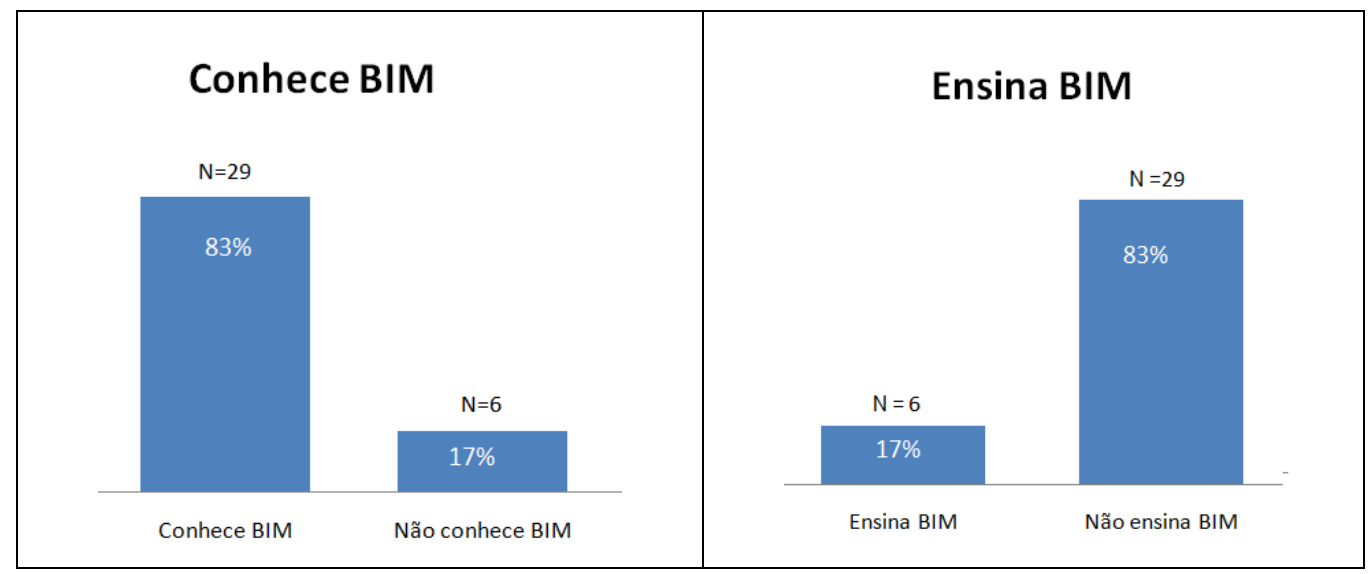

Fonte: Os autores, 2015 
Entre os professores que conhecem BIM, a maioria não forneceu uma definição para o termo $(34 \%, \mathrm{~N}=10)$ ou definiu BIM como um sistema, ferramenta ou plataforma $(28 \%, \mathrm{~N}=8)$. Esses dados podem ser visualizados na Figura 3.

Figura 3 - Definições de BIM na percepção dos professores

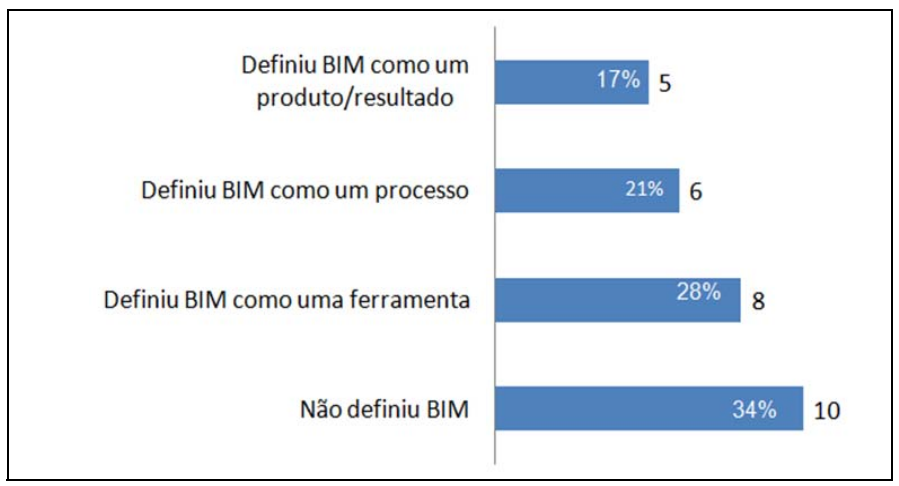

Fonte: Os autores, 2015

De acordo com as respostas dos professores, BIM está sendo ensinado em disciplinas de: 'Transportes', 'Introdução ao Projeto na Engenharia', 'Representação Gráfica Digital' e 'Topografia'. Entretanto, existe também a possibilidade de que BIM seja ensinado em disciplinas de: 'Tecnologia de Construção', 'Sistemas Prediais' e 'Gestão da Construção'.

Na percepção dos professores, algumas modificações poderiam ser efetuadas no currículo. Por exemplo:

(a) a disciplina 'Tecnologia de Construção' para Arquitetura poderia ser reestruturada em termos de metodologia, exercícios e avaliação;

(b) a disciplina 'Computação Gráfica' para arquitetura poderia preparar o aluno para cursar uma disciplina BIM, que, atualmente, é optativa, mas que, no futuro, será obrigatória;

(c) na disciplina 'Sistemas Prediais' para Engenharia Civil, os alunos poderiam desenvolver projetos em BIM;

(d) a disciplina 'Topografia' para Engenharia Civil poderia aproveitar um tutorial que já está sendo desenvolvido para aplicar BIM;

(e) a disciplina 'Transportes' para Engenharia Civil introduziria BIM para integrar informações representadas em GIS e CAD, ou até para compatibilizar as informações de softwares alternativos de CAD ou GIS;

(f) uma disciplina de projeto poderia ser integrada à disciplina 'Conforto Ambiental 6', na qual os alunos fazem uma análise de desempenho e propõem o redesenho de um edifício; e

(g) a disciplina 'Representação Gráfica Digital' para Engenharia Civil poderia ensinar uma ferramenta BIM.

$\mathrm{Na}$ opinião dos professores, seriam necessários recursos para a implementação de BIM, como por exemplo: o apoio de colegas do Departamento, o apoio do Departamento, o apoio de revendedores de software e de empresas, além da necessidade de efetuar modificações nas ementas das disciplinas, em termos de conteúdos e metodologia.

Quanto ao ano do currículo em que BIM deve ser introduzido, a maioria dos professores, que leciona para Engenharia Civil, que corresponde a $44 \%(\mathrm{~N}=7)$, prefere o primeiro ano, 


\section{Q1}

enquanto que a maioria dos professores, que leciona para Arquitetura, que corresponde a $32 \%(\mathrm{~N}=5)$, sugere o $1^{\circ}$ e o $3^{\circ}$ ano, conforme pode ser observado na Figura 4.

Figura 4 - Ano do currículo em que BIM deve ser introduzido

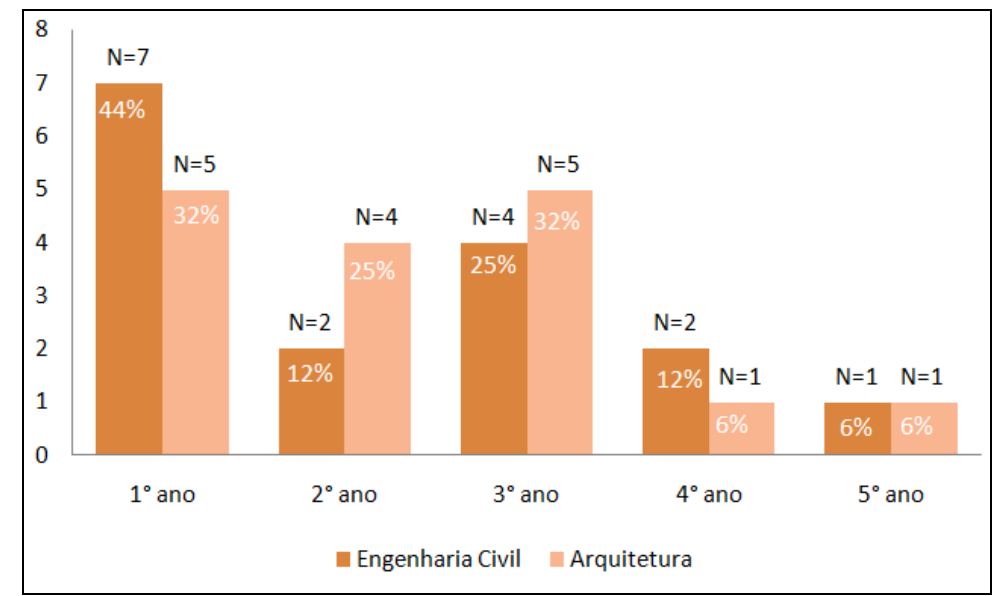

Fonte: A autora, 2014

Quanto aos obstáculos para introduzir BIM no currículo, a maioria dos professores considera que é pouco ou nada verdadeiro que: (a) o uso de ferramentas BIM em projeto prejudica o pensamento criativo; (b) é difícil saber qual ferramenta BIM será adotada no futuro; (c) o mercado de trabalho ainda não está requerendo especialistas BIM; (d) as escolas precisam ver para crer antes de introduzir BIM; (e) a política de TI da escola inviabiliza o uso de um servidor BIM; e (f) faltam bons computadores e equipamentos apropriados.

Por outro lado, a maioria dos professores percebeu que é totalmente verdadeiro e bastante verdadeiro, que: (a) existe falta de relacionamento curricular entre projeto e gestão; (b) há dificuldades para integrar disciplinas do currículo; e (c) há falta de 'espaço' no currículo para incluir novas disciplinas.

Os respondentes concordam, em parte, que: (a) falta uma metodologia específica para o ensino de BIM; (b) há indisposição de professores para ensinar BIM; (c) falta de tempo dos professores para planejar uma disciplina BIM; (d) órgão de certificação não exige habilidades BIM; (e) faltam materiais didáticos sobre BIM; (f) há problemas com software BIM e hardware; $(\mathrm{g})$ há dificuldade para conciliar horários; e $(\mathrm{h})$ há falta de vontade para mudar o currículo. Esses dados podem ser observados no gráfico da Figura 5. 


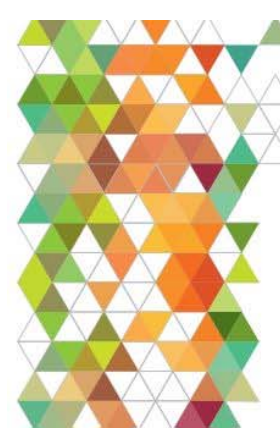

VII Encontro de Tecnologia de Informação

e Comunicação na Construção

Edificações, Infra-estrutura e Cidade: Do BIM ao CIM

ufpe.br/tic2015

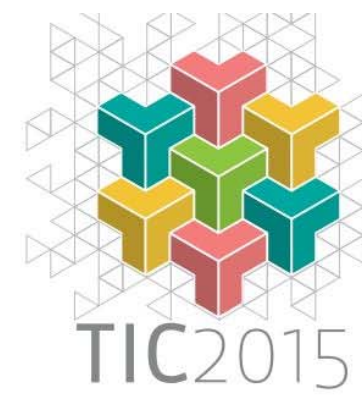

Figura 5 - Percepções quanto a obstáculos para introduzir BIM no currículo

Usar ferramenta BIM em projeto pode prejudicar o pensamento criativo

Politica de TI da escola inviabiliza o uso de um servidor BIM

Falta de relacionamento curricular entre Projeto e Gestão

O mercado de trabalho ainda não demanda especialistas BIM

Dificuldade para conciliar horários de turmas interdisciplinares

Não se sabe qual software BIM será adotado pela indústria no futuro

Problemas com software BIM e hardware

Falta de bons computadores e equipamentos apropriados

Falta de materiais didáticos específicos sobre BIM

Órgãos de certificação ainda não exigem habilidades em BIM

Dificuldade para integrar disciplinas do currículo

Falta de tempo dos professores para planejar uma disciplina BIM

Falta de 'espaço' no currículo para incluir novas disciplinas

Falta de vontade para mudar o currículo

Indisposição dos professores para aprender e/ou ensinar BIM

Faltam professores capacitados emBIM

Ceticismo da escola (primeiro quer ver para crer)

Falta de uma metodologia específica para o ensino de BIM
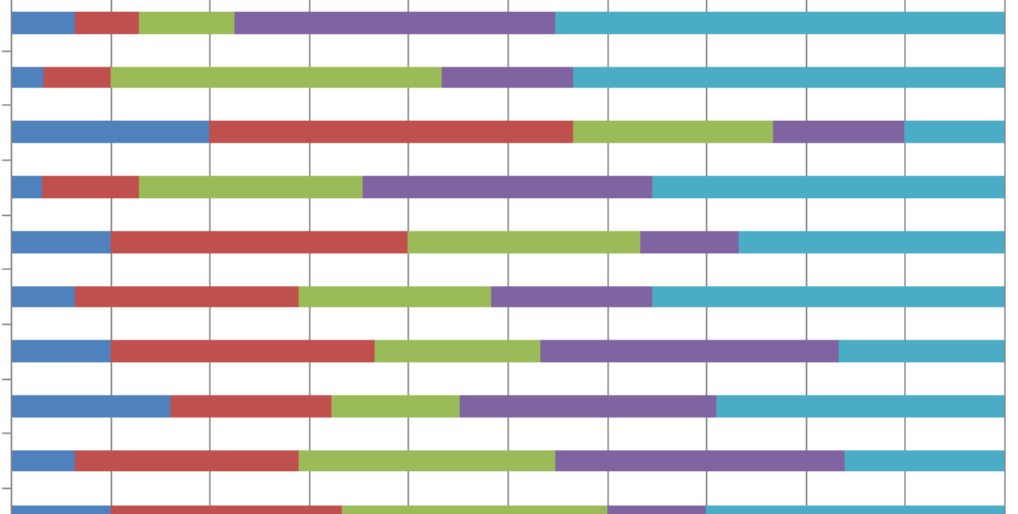

- Totalmente Verdadeiro

Bastante verdadeiro

- Médio

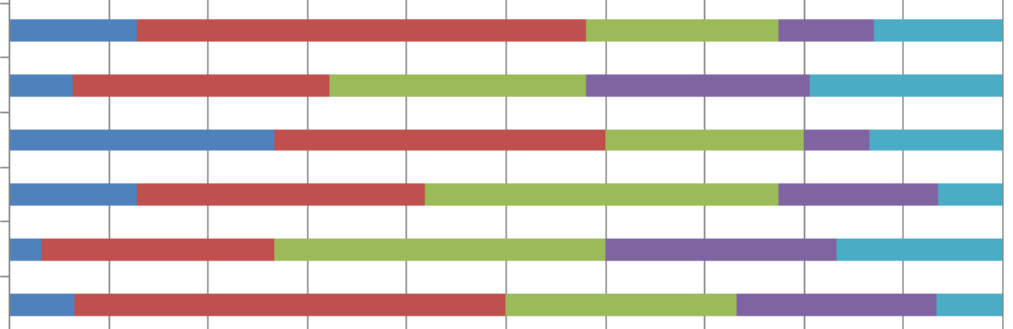

- Pouco verdadeiro

Nada verdadeiro

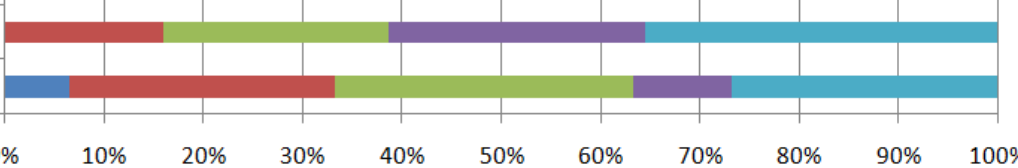

Fonte: A autora, 2014 


\section{PROPOSTA PARA A INTRODUÇÃO DE BIM NOS DOIS CURRÍCULOS}

No curso de Arquitetura, recomenda-se ensinar ferramentas BIM na disciplina 'Computação Gráfica' e introduzir conteúdos BIM em disciplinas de 'Tecnologia de Construção', uma vez que estas áreas estão, diretamente, relacionadas ao BIM e existe interesse por parte de alguns professores dessas disciplinas.

A disciplina 'Prática de Empreendimentos na Área de Edificações', que, atualmente, ensina alguns tópicos BIM, poderia se tornar uma disciplina BIM específica, e, para cursá-la, os alunos deveriam ter concluído a disciplina 'Computação Gráfica', pela qual eles obteriam conhecimentos e habilidades na ferramenta BIM.

A disciplina 'Conforto Ambiental 6' poderia ser integrada a uma disciplina de Projeto, de forma que o aluno pudesse desenvolver um projeto e, ao mesmo tempo, efetuar algumas análises.

Antes de defenderem o projeto de conclusão de curso, os alunos poderiam cursar, no quarto ano, uma nova disciplina eletiva, intitulada 'Ateliê BIM de Colaboração', que, por sua vez, poderia ser integrada à disciplina 'Prática de Empreendimentos na Área de Edificações'.

No curso de Engenharia Civil, ferramentas BIM poderiam ser ensinadas em 'Representação Gráfica Digital', para que os estudantes adquirissem, logo no início do curso, uma base no domínio da ferramenta. Um exercício de aplicação da ferramenta BIM poderia ser realizado na disciplina 'Topografia', uma vez que existe interesse do docente desta disciplina no que se refere ao desenvolvimento de tutoriais.

Aos alunos, devem ser disponibilizadas oportunidades para que possam desenvolver seus projetos utilizando ferramentas BIM. Um exemplo são os projetos de 'Sistemas Prediais', pois existe interesse por parte dos professores dessas disciplinas quanto ao ensino de BIM.

A disciplina de 'Transportes' poderia ser reformulada para introduzir conteúdos BIM, com o objetivo de ensinar aos alunos a integração de informações representadas em CAD e GIS.

Além disso, poderia ser criada uma nova disciplina eletiva, intitulada 'Ateliê BIM de Colaboração', também ofertada para alunos de Arquitetura e integrada com a disciplina eletiva: 'Gestão do Processo de Projeto'. Dessa forma, os alunos de engenharia civil aprenderiam conceitos e usos de BIM, além de desenvolverem um projeto em equipe interdisciplinar.

Nos $3^{\circ}$ e $4^{\circ}$ anos, poderia ser criada uma disciplina obrigatória intitulada 'Ateliê de Integração', preferencialmente no segundo semestre. Nesta disciplina, os alunos desenvolveriam um projeto BIM com os conhecimentos adquiridos nas disciplinas ofertadas durante o ano. Esta proposta de modificação para os dois currículos pode ser visualizada nas Figuras 6 e 7. 
Figura 6 - Disciplinas com potencial BIM e proposta de nova(s) disciplina(s) para o curso de Arquitetura e

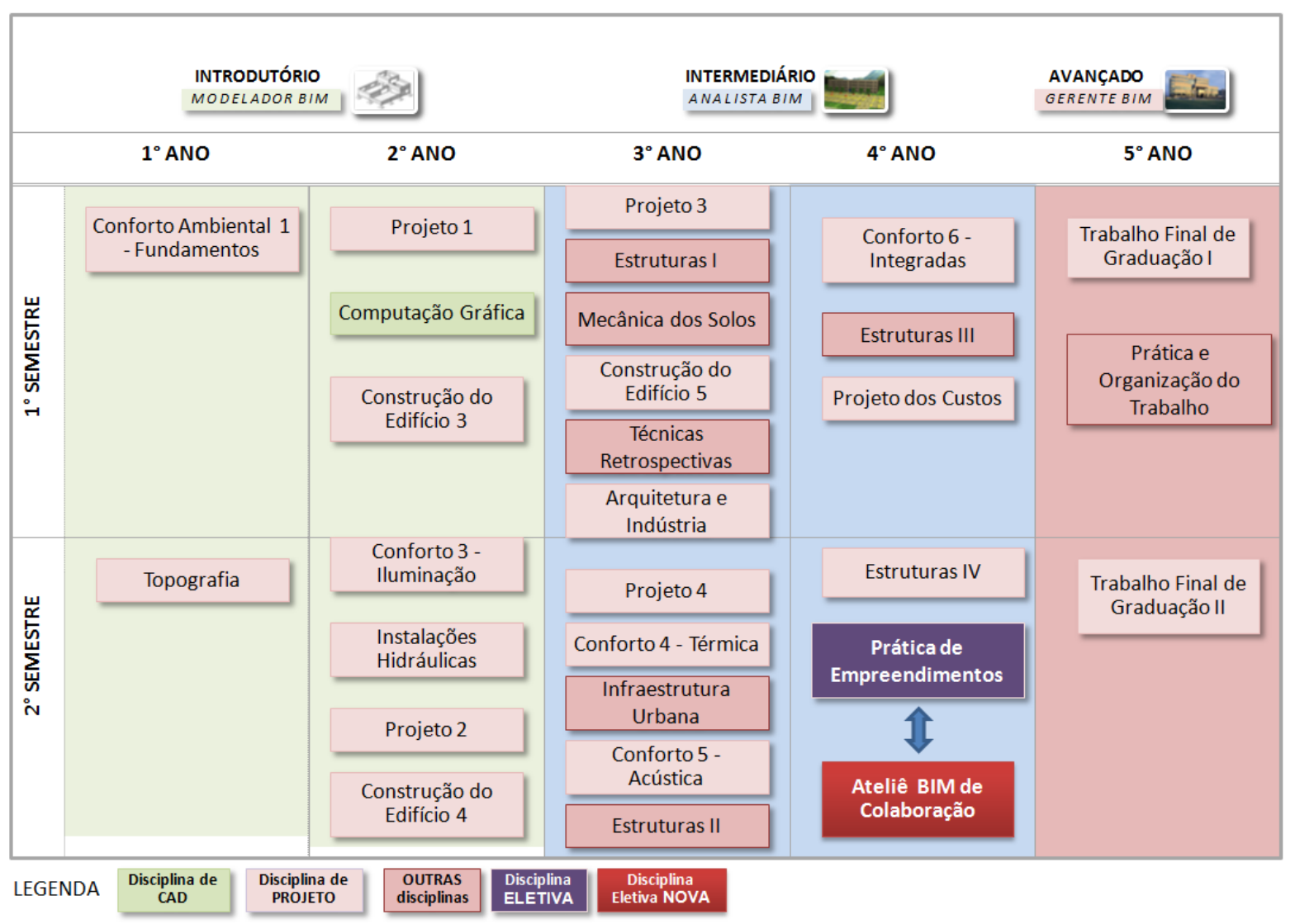

Fonte: Os autores, 2015 
Figura 7 - Disciplinas com potencial BIM e proposta de nova(s) disciplina(s) para o curso de Engenharia Civil

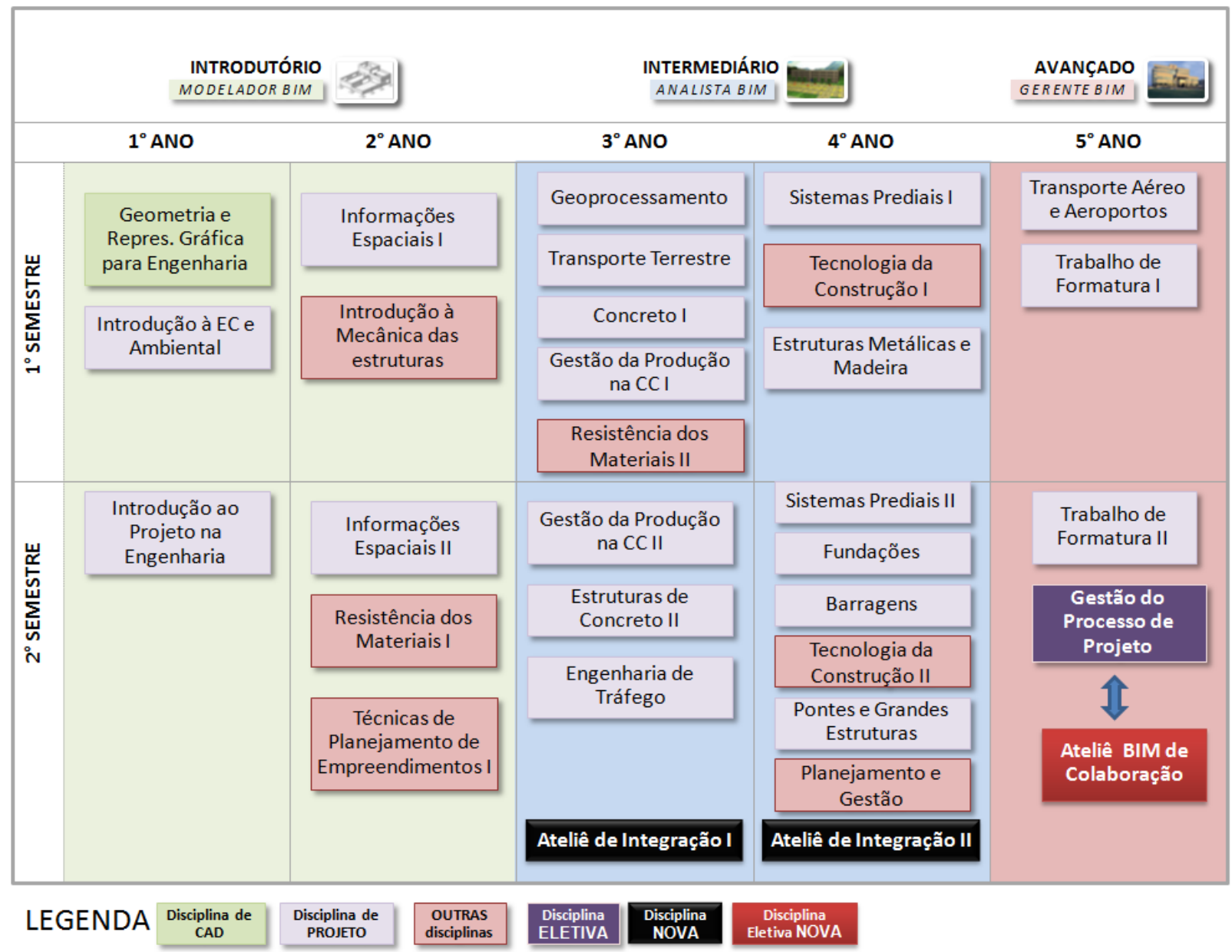

Fonte: Os autores, 2015

\section{CONCLUSÕES}

O primeiro passo para introduzir BIM no currículo é saber o que professores pensam com relação ao BIM, por exemplo, suas percepções quanto ao significado de BIM e às dificuldades e obstáculos que encontrariam ou encontraram para implantar BIM em suas disciplinas, e analisar suas sugestões.

Nos currículos analisados a maioria dos professores apontou como obstáculos: a falta de integração de disciplinas do currículo, principalmente, nas áreas de Projeto e Gerenciamento da Construção, e a falta de 'espaço' no currículo para incluir novas disciplinas. Com relação a estes maiores problemas apontados pelos professores, possíveis soluções seriam: identificar as disciplinas do currículo com potencial para introduzir BIM, discutir o currículo com os professores e alunos e fazer uma proposta de ensinar BIM em disciplinas existentes do currículo.

Com este estudo, verificamos também que, para implementar BIM no currículo, também é importante: (a) capacitar professores por meio de palestras, workshops e cursos fora da grade horária para, principalmente, ensinar conceitos e usos de BIM; (b) desenvolver conteúdos didáticos relacionados ao BIM em projetos de iniciação científica; (c) desenvolver módulos de ensino de BIM em parceria com empresas de AECO; (d) promover projetos de pesquisa sobre BIM; (e) divulgar a importância e vantagens de BIM para o setor de AECO; 
(f) capacitar técnicos de laboratório para auxiliarem o professor nas aulas práticas; (f) promover treinamentos para monitores auxiliarem o professor em sala de aula; e (g) buscar o apoio de revendedores de software e de empresas de AECO, no sentido de proporcionar palestras e esclarecer dúvidas relacionadas a BIM.

Neste estudo, foram apresentadas algumas sugestões, porém, cabe ao docente da disciplina determinar quais habilidades BIM ele/ela pretende desenvolver nos alunos.

\section{AGRADECIMENTOS}

O segundo autor agradece ao apoio do CNPq - Conselho Nacional de Desenvolvimento Científico e Tecnológico e da Fundação de Amparo à Pesquisa do Estado de São Paulo (FAPESP).

\section{REFERÊNCIAS}

AHN, Y. H., CHO, C. S., LEE, N., Building Information Modeling: Systematic Course Development for Undergraduate Construction Students. Journal of Professional Issues in Engineering Education and Practice. 2013. 139 (4), p. 290-300.

BARISON, M. B. Introdução de Modelagem da Informação da Construção (BIM) no Currículo: uma contribuição para a formação do projetista / M.B. Barison. - versão corr. -- São Paulo, 2015. 387 p. Tese (Doutorado) - Escola Politécnica da Universidade de São Paulo. Departamento de Engenharia de Construção Civil.

BERNSTEIN, P. G. BIM: Practice Context and Implications for the Academy. In: DEAMER, P.; BERNSTEIN, P. G (Ed). BIM in Academia. New Haven: Yale School of Achitecture, 2011. p. 4-10.

CLEVENGER, C.M., et al. Integrating BIM into Construction Management Education. In: THE BIMRELATED ACADEMIC WORKSHOP, 2010, Washington D.C. Proceedings...Washington D.C.: SALAZAR, G.; ISSA, R. (Ed.), 2010. Disponível em:

$<$ http://www.buildingsmartalliance.org/client/assets/files/bsa/bsa conference proceedings 1210.pdf>. Acesso em: 2 mai. 2011.

COOKSEY, J. M.; SCHIFF, S. D. Integration of Building Information Modeling into a Civil Engineering Curriculum. In: 2012 ASEE SOUTHEAST SECTION CONFERENCE. 2012, Starkville. Proceedings... Starkville: SPSU. Disponível em: <http://155.225.14.146/asee-

se/proceedings/ASEE2012/Papers/FP2012c00119 529.PDF>. Acesso em: 19 ago. 2012.

GAO, Z.; MCENTYRE, C.; GE, Z. Integrating BIM in Teaching Project Scheduling and Control. In: $119^{\text {th }}$ ASEE ANNUAL CONFERENCE EXPOSITION, 2012, San Antonio Proceedings... San Antonio: ASEE. Disponível em: <http://www.asee.org/public/conferences/8/papers/4868/view>. Acesso em: mai. 2013.

GORDON, C.; AZAMBUJA, M.; WERNER, A. M. BIM across the Construction Building. In: 2009 ASC REGION III CONFERENCE, 2009, Illinois. Proceedings... Illinois: ASC.

Disponívelem:<http://region3.ascweb.org/Documents/ASC R3 09 Papers/Proceeding\%20of\%20the \%202009\%20ASC3\%20Regional\%20Conference-2009-final.pdf>. Acesso em: 22 jun. 2011.

LEE, N; HOLLAR, D. A. Probing BIM Education in Construction Engineering and Management Programs Using Industry Perceptions. In: $49^{\text {th }}$ ASC ANNUAL INTERNATIONAL CONFERENCE, 2013, San Luis Obispo. Proceedings... San Luis Obispo: ASC/Cal Poly. Disponível em: <http://ascpro.ascweb.org/chair/paper/CEUE44002013.pdf>. Acesso em: 11 mai. 2013.

LUCAS, J. Deriving learning outcomes for BIM implementation into the CSM curriculum based on Industry Expectation. In: $50^{\text {th }}$ ASC ANNUAL INTERNATIONAL CONFERENCE, 2014, Washington D. C. Proceedings...Washington D. C: ASC, 2014. 
MACDONALD, J. A. Framework for collaborative BIM education across the AEC disciplines. In: $37^{\text {th }}$ ANNUAL CONFERENCE OF THE AUSTRALASIAN UNIVERSITIES BUILDING EDUCATORS ASSOCIATION, 2011, Sydney. Proceedings... Sydney: AUBEA/ UNSW, 2011.

Disponívelem:<http://uts.academia.edu/JenniferMacdonald/Papers/1779185/A FRAMEWORK FOR COLLABORATIVE BIM EDUCATION ACROSS THE AEC DISCIPLINES>. Acesso em: 22 ago. 2012.

MANDHAR, M.; MANDHAR, M. Biming the architectural curricula - integrating building information modeling (BIM) in architectural education. International Journal of Architecture, v.1, n. 1, 2013. p. 01-20.

MUTAI, A.; GUIDERA, S. Building Information Modeling: the implications of government and industry initiatives for AEC Education. In: ASEE 2008 AMERICAN SOCIETY ENGINEERING EDUCATION CONFERENCE, 2008, Pittsburgh. Proceedings... Pittsburgh: ASEE, 2008. Disponível em: <http://soa.asee.org/paper/conference/paper-view.cfm?id=7884>. Acesso em: 13 nov. 2009.

NEJAT, A.; DARWISH, M. M.; GHEBRAB, T. BIM Teaching strategy for Construction Engineering Students.In: 2012 ASEE ANNUAL CONFERENCE AND EXPOSITION, 2012, San Antonio. Proceedings.... San Antonio: ASEE. Disponível em: <http://www.asee.org/public/conferences/8/registration/sessions?page=6>. Acesso em: ago. 2012.

PIKAS, E.; SACKS, R.; HAZZAN, O. Building Information Modeling Education for Construction Engineering and Management. II: Procedures and Implementation Case Study. Journal of Construction Engineering and Management. v. 139 (11), 2013.

SACKS, R.; PIKAS, E. Building Information Modeling Education for Construction Engineering and Management. I: Industry Requirements, State of the Art, and Gap Analysis. Journal of Construction Engineering and Management. v. 139, n 11, 2013.

SCHMELTER, S.; CORY, C. Comercial Construction BIM course created for academia. JBIMJournal of Building Informacion Modeling, Washington, p. 40-41, Spring 2009. Disponível em: $<$ http://www.wbdg.org/pdfs/jbim spring09.pdf>. Acesso em: 10 jun. 2009.

TAYLOR, J. M.; SATTINENE, A. BSCI 5970/6970: Special topics -Building Information Modeling. 2007. Disponível em:

<http://www.cadc.auburn.edu/BSCI/FacStaff/files/Syllabii/Sattineni/BSCI\%205970-6970/BIM.pdf>. Acesso em: 20 mar. 2009.

WONG, K.; WONG, K.; NADEEM, A. Building Information Modeling for tertiary construction education in Hong Kong. Journal of Information Technology in Construction. v. 16, p. 467-476, 2011. Disponível em: <http://www.itcon.org/2011/27>. Acesso em: 20 fev. 2012.

WU, W.; ISSA, R. R. A., BIM Education for new careers options: an initial investigation. In: BIM ACADEMIC WORKSHOP 2013, Washington, D. C. 2013a. Proceedings... Washington, D. C., buildingSMART Alliance. 2013a. Disponível em:

<http://www.projects.buildingsmartalliance.org/files/?artifact id >. Acesso em: 22, mai. 2013. 